\title{
The development of prehospital emergency medical services (PEMS) in China
}

\section{Z. DING}

Emergency Department, Chinese PLA General Hospital and Post-graduate Medical School, Beijing

Prehospital emergency medical services (PEMS) in China have developed in the following ways:

(1) Well equipped Emergency Medical Centres (EMC), organized independently such as Beijing EMC or affiliated to big hospitals such as Chongqing EMC, with primary stations in local regions.

(2) Some well-equipped emergency clinics in a big hospital in charge of prehospital care such as in Guangzhou city. The Chinese PLA General Hospital has organized PEMS for more than 20 years. A team of a physician, nurse and technician are on call to provide on-site resuscitation and rapid transport of patients with constant monitoring.

With the advancement of science and technology, the increase in industry and traffic and the prolongation of people's lives, the incidence of heart disease, cerebro vascular accidents and injuries have increased in recent years in China. The Emergency Medical Service System faces the problem of providing rescue, on site resuscitation and transport of patients with monitoring. Good PEMS is the key to the reduction of mortality, the number of sudden deaths and other life threatening conditions which are daily occurrances.

Emergency medicine is a new branch of medicine in China. The prehospital care (PHC) is deficient. The county hospitals dispatch ambulances, mostly for transport of patients rather than their immediate medical resuscitation. Only in the past 2 or 3 years, following the development of emergency medicine as a whole, has PEMS in China begun to develop. Two types of PHC have been built up. The first type are well equipped Emergency Medical Centres (EMC). Some of which are organized independently, such as Beijing EMC, with a dispatching office, special telepone line ('120') and rescue staff consisting of experienced physicians, surgeons, well trained nurses, technicians, and 60 ambulances containing resuscitation and monitoring equipment. This Centre coordinates with primary stations in 7 local regions of the city. About $\mathbf{4 0}$ hospitals accept the patients that are transferred to them. Coordination between the EMC and regional stations needs strengthening. 
Some EMC's are affiliated to large hospitals such as Chongqing EMC, which is attached to the 4 th hospital of the city. This EMC has a dispatching office rescue groups and it arranges for large and medium hospitals to accept patients. Yet there is still muchộ to be done in organizing an efficient network that transports and delivers patients promptly.

The second type of PHC consists of well-equipped emergency clinics in big hospitals $\underset{\vec{\rho}}{\overrightarrow{2}}$ which are in charge of PHC such as in Guangzhou city. Twenty hospitals take charge ofo PEMS in 8 regions, forming a network. By means of telephone, radio transmitter and $\frac{\bar{N}}{\bar{N}}$ command car, the officer of the public health bureau can dispatch rescue groups to the scene more promptly, by shortening the running radius.

Chinese PLA General Hospital has taken responsibility for PEMS for more than 20 क years. Teams of one physician, one nurse and one technician are on call, then go to the $\vec{\circ}$ scene and transport resuscitated and monitored patients to the hospital. The service is $\overrightarrow{\vec{\omega}}$ limited to members of our system and their families.

Two groups of PHC cases were compared and analysed as follows:

Group A (GA) from 1981-1985 230 cases

Group B (GB) from 1986-1988 100 cases

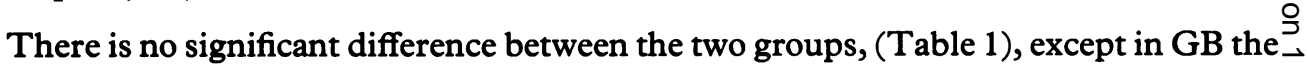
mean age is much older. The two groups are comparable.

\section{Comparison of the two groups}

Time in dispatching rescue group.

The shortening of dispatching time was significant, suggesting improvements in the

Table 1 General Data

\begin{tabular}{lccc}
\hline & GA & GB & P \\
Average age & $62 \cdot 91 \pm 7.0$ & $66.37 \pm 0.92$ & 0.01 \\
\hline No. of cases & & & \\
male & 186 & 87 & $>0.05$ \\
female & 44 & 13 & $>0.05$ \\
acute myocardial infarction* & 60 & 32 & \\
CHD (non-AMI) & 96 & 36 & \\
Sudden death & 22 & 17 & \\
Others & 52 & 15 & \\
\hline
\end{tabular}

*No significant difference in incidence, sites and complications of acute myocardial infarction between two groups (P 0.05)

Table 2 Time in dispatching

\begin{tabular}{|c|c|c|c|}
\hline \multirow[b]{2}{*}{ Time } & \multicolumn{2}{|c|}{ Average time (min.) } & \multirow[b]{2}{*}{$\mathbf{P}$} \\
\hline & Group A & Group B & \\
\hline dispatching ambulance & $10.71 \pm 0.57$ & $8.86 \pm 0.35$ & $<0.01$ \\
\hline arrival of rescue groups & $16.79 \pm 0.98$ & $14.61 \pm 0.68$ & $>0.05$ \\
\hline
\end{tabular}


organization of the rescue groups and in communication with the basic emergency stations. There was no significant change in arrival time. This was due to the fact that rescue of some patients required travel of long distances. The difference between 'system' and 'service' is a challenge we face.

\section{Mortality}

Only patients with AMI, alive on arrival of the rescue group were analysed in Table 4.

\section{Sudden death}

The number of cases who died before arrival of the rescue team.

Admission rate in cases of Non-AMI CHD (coronary heart disease)

These patients had unstable angina or CHD with other complications.

The development of PEMS in China does not progress evenly and is not yet well distributed. We must work hard in constructing a better network and especially emphasize the need to improve the primary ambulance stations and PEMS in the weaker regions.

Table 3 Mortality

\begin{tabular}{lcccc}
\hline \multirow{2}{*}{ Period } & \multicolumn{2}{c}{ No. of deaths } & \multicolumn{2}{c}{ Mortality } \\
& GA (60) & GB (32) & GA & GB \\
\hline Before arriving the hospital & 4 & 1 & 6.67 & $3 \cdot 17$ \\
On discharge & 12 & 5 & $21 \cdot 43$ & $16 \cdot 13$ \\
Total & 16 & 6 & 26.67 & 18.75 \\
\hline
\end{tabular}

GB in prehospital period and on discharge, mortality decreased, but not significantly $P>0.05$.

Table 4 Sudden death

\begin{tabular}{lcccccc}
\hline & \multicolumn{3}{c}{ No. of cases } & \multicolumn{2}{c}{ Percentage \% } & \\
& GA (230) & GB (100) & GA & GB & P \\
\hline Sudden death & 22 & 11 & 9.56 & 11.0 & $\begin{array}{c}>0.05 \text { No signi- } \\
\text { ficant difference }\end{array}$ \\
\hline
\end{tabular}

There is a need to popularize CPR, raise the quality of medical personnel in basic stations and reduce travelling times.

Table 5 Admission rate

\begin{tabular}{lcccccc}
\hline \multirow{2}{*}{ Disease } & \multicolumn{3}{c}{ No. of admission } & \multicolumn{3}{c}{ Percentage \% of admission } \\
& GA (96) & GB (36) & GA & GB & \\
\hline Non-AMI CHD & 37 & 24 & 38.54 & 66.67 & $<0.01$ \\
\hline
\end{tabular}




\section{DISCUSSION}

We suggest that adequate PEMS needs the following key points:

(1) Organization and management to ensure PHC based on clear rules, a good system. and high standards.

(2) Quality assurance in PHC personnel must be maintained by strict and repeatedo training in emergency medical theories and practice, including the use of modern emergency equipment.

(3) Advanced system of communication and ambulances with a variety of resuscitation $\stackrel{\mathbb{D}}{\circ}$ and monitoring systems to provide a 'mobile hospital'.

(4) Basic education for the public in CPR to make rescue more efficient.

\section{ACKNOWLEDGEMENTS}

The author wishes to express kind acknowledgement to Dr Frank Garlic, Committee $\stackrel{\circ}{\circ}$ Member of the 2nd International Conference on Emergency Medicine, for his con-? structive criticism and suggestions.

\section{REFERENCES}

Huang Shijia \& Ouyang Binghui (1986) Discussion on the administration patterns and characteristics of rescue organization in Guangzhou city. Proceedings of The First Chinese Symposium on Emergency Medicine 6-9.

Shao Xiaohong (1987) The development of EMSS in China. Proceedings of International Urban EMS Symposium 1-6, Beijing, China. 